The Children's Headache Assessment Scale (CHAS), a parent questionnaire focusing on situations and events surrounding the headaches, emphasizes stress antecedents and coping responses rather than symptom details, and is of value in following responses to behavioral therapy. Environmental factors, including nutrition, are important in the etiology and management of childhood headache and warrant further study. (see Progress in Pediatric Neurology I, 1991, pp141-150).

\title{
ACUTE CONFUSIONAL MIGRAINE
}

Of 76 children admitted with migraine between 1982 and 1990, 13 had a discharge diagnosis of confusional migraine at British Columbia Children's Hospital, Vancouver, Canada. A retrospective analysis of cases showed a preponderance of males to females (11:2), age range of 6 to 15 years (mean, 10 years), all having headache followed by a period of confusion, lasting 2-24 hours, and 4 having recurrent episodes. Mild head trauma preceded the headache in 4 patients. In addition to confusion, agitation occurred in 8 patients, past history of headache in 7, and a family history of migraine in 10 . One of 11 patients with CT scans had an arachnoid cyst. EEG was mildly abnormal in 2 of 4 patients with recordings. CSF was normal in 2 patients studied. (Shaabat A. Confusional migraine in childhood. Pediatr Neurol July 1996;15:23-25). (Respond: Dr Shaabat, Dept Pediatrics, College of Medicine and Allied Health Sciences, King Abdulaziz University, PO Box 6615, Jeddah 21452, Saudi Arabia).

COMMENT. A diagnosis of migraine should be considered in children with episodes of acute confusion and agitation, lasting from 1 to 24 hours, preceded by headache and sometimes, mild head trauma, and a positive family history of migraine. It is interesting that CT scans uncovered an arachnoid cyst in one patient, a space-occupying lesion, usually developmental in origin, and known to be complicated by headaches and seizures in some. Further studies of the EEG, obtained in only 4 of the above patients, and response to therapy would be of interest.

\section{NEURODEGENERATIVE DISORDERS}

\section{DECIDUOUS TEETH IN TUBEROUS SCLEROSIS}

The diagnostic significance of enamel pits in shed deciduous teeth from 20 patients with tuberous sclerosis was investigated at the University of Copenhagen, Denmark. Examination with a surface microscope found enamel pits in all 87 teeth obtained from 20 tuberous sclerosis patients, but none in 253 deciduous teeth from 142 patients with cerebral palsy, phenylketonuria and Down syndrome, as well as healthy controls. The facial surfaces of the central incisor, lateral incisor and canine teeth were most frequently affected. (Russell BG, Russell MB, Praetorius F, Russell CA. Deciduous teeth in tuberous sclerosis. Clin Genet July 1996;50:36-40). (Respond: Dr Bjorn G Russell, Copenhagen County Dental Clinic for Handicapped, Bank Mikkelsens Vej 1, 2820 Gentofte, Denmark).

COMMENT. The occurrence of enamel pits in deciduous and permanent teeth may be a useful diagnostic criterion for tuberous sclerosis. Examination of the labial surfaces of the cleaned central and lateral incisors and canine teeth, using a magnifying glass, may be as important as the search for hypopigmented macules on the skin. 